\title{
Erratum
}

\section{Spectral trends in planetary nebulae: The roles of radiative and shock excitation}

\section{J.P. Phillips and V. Guzman}

Instituto de Astronomia y Meteorologia, Av. Vallarta 2602, Col. Arcos Vallarta, C.P. 44130 Guadalajara, Jalisco, Mexico

Astron. Astrophys. Suppl. Ser. 130 (1998) 465-476

In the paper "Spectral trends in planetary nebulae: The roles of radiative and shock excitation" we make passing reference to precessing jets within NGC 6543. Dr. L. Miranda has kindly pointed out two prior mentions of possible precession in this source, by Miranda \& Solf (1992) and Balick \& Preston (1987). We are happy to acknowledge these previous analyses.

Balick B., Preston H.L., 1987, AJ 94, 958

Miranda L.F., Solf J., 1992, A\&A 260, 397 